\title{
Impactos na saúde mental dos profissionais de saúde atuantes na linha de frente a pandemia do COVID-19
}

Impacts on mental health of health professionals working on the front line of the COVID-19

pandemic

Impactos en la salud mental de los profesionales de la salud que trabajan en la primera línea de la pandemia de COVID-19

Maria Eduarda Wanderley de Barros Silva ORCID: https://orcid.org/0000-0002-4642-3282 Universidade Federal de Campina Grande, Brasil E-mail: eduarda.wanderley@outlook.com

Thaís Andrade dos Santos ORCID: https://orcid.org/0000-0002-9669-7468 Universidade Federal de Pernambuco, Brasil E-mail: thais.andradesantos@ufpe.br

Vivian Marinho da Silva ORCID: https://orcid.org/0000-0001-8150-9754 Universidade Federal de Pernambuco, Brasil E-mail: vivian.marinho@ufpe.br

Juliana Alves de Siqueira ORCID: https://orcid.org/0000-0002-1441-9676 Universidade Federal de Pernambuco, Brasil E-mail: juliana.asiqueira@ufpe.br Yasmin Bezerra Ribeiro ORCID: https://orcid.org/0000-0002-2658-7271 Universidade Federal de Pernambuco, Brasil E-mail: yasmin.bezerraribeiro@ufpe.br

Bárbara Letícia Sabino Silva ORCID: https://orcid.org/0000-0003-0610-0419 Universidade Federal de Pernambuco, Brasil E-mail: barbaralsabinos@gmail.com

Conceição Aparecida Silva Sousa ORCID: https://orcid.org/0000-0002-6640-2841 Universidade Federal de Pernambuco, Brasil E-mail: conceicao.sousa@ hotmail.com

Mharianna Phaitter Silva Arruda ORCID: https://orcid.org/0000-0002-7682-6410 Universidade Federal de Pernambuco, Brasil E-mail: mhariannapsa@gmail.com

Maria Letícia Cardoso da Silva Barbosa ORCID: https://orcid.org/0000-0002-2935-6882 Universidade Federal de Campina Grande, Brasil

E-mail: marialeticia20151@ hotmail.com

Fernanda Félix de Araújo Varela ORCID: https://orcid.org/0000-0001-6550-9113 Universidade Federal de Campina Grande, Brasil E-mail: fernandafeliix@ @otmail.com

Maria Conceição Diniz Teixeira ORCID: https://orcid.org/0000-0001-7055-3266 Universidade Federal do Rio Grande do Norte, Brasil E-mail: maria.diniz.115@ufrn.br

Rubens de Andrade Martins ORCID: https://orcid.org/0000-0002-2513-9864 Universidade Tiradentes, Brasil

E-mail: rubensdeandrade@ @otmail.com 
Thalita Emília Correia Arruda

ORCID: https://orcid.org/0000-0001-9426-0142 Universidade Maurício de Nassau, Brasil E-mail: thalitaemiliacontato@gmail.com

Dulcineide Maria da Silva

ORCID: https://orcid.org/0000-0002-2823-9131

Centro Universitário UNIFBV, Brasil

E-mail: dulcineidesilvacontato@gmail.com

Larissa Lima Soares

ORCID: https://orcid.org/0000-0002-7682-372X

Centro Universitário Tiradentes, Brasil

E-mail: larissalyma@hotmail.com

\begin{abstract}
Resumo
Trata-se de uma revisão integrativa da literatura de abordagem qualitativa. Sendo seguida as seguintes etapas de identificação do tema e seleção da pesquisa, elaboração dos critérios de exclusão e inclusão, seleção dos estudos categorização dos estudos selecionados, análise e interpretação dos resultados. A pesquisa teve como pergunta norteadora a seguinte questão: "Quais os impactos a pandemia do COVID-19 trouxeram na saúde mental dos profissionais atuantes na linha de frente?”. Os estudos destacam as consequências da pandemia do covid-19 na vida dos profissionais de saúde, ao apontar que a exposição a uma situação de emergência em saúde pública provocou risco ocupacional e sofrimento psicológico devido ao trauma, desvalorização profissional, auto isolamento e quarentena. Com isso, foi desencadeado diversos impactos negativos na saúde mental dos profissionais manifestando-se mediante a sintomas de insônia, angústia, ansiedade, depressão, medo, frustração e estresse pós-traumático. Conclui-se que, os impactos que a pandemia trouxe com a chegada de um novo vírus pouco conhecido, atingiu diretamente na sociedade e refletiu nos profissionais da saúde que atuam na linha de frente, contudo, o apoio e o acolhimento psicológico poderão contribuir para encarar os efeitos da pandemia na saúde mental, além de outras alternativas como cursos de capacitações para orientar a equipe de saúde no tratamento e enfrentamento de problemas psicológicos.
\end{abstract}

Palavras-chave: Coronavírus; Saúde mental; Pessoal da saúde.

\begin{abstract}
This is an integrative review of the qualitative approach literature. The following steps were followed: identification of the theme and selection of the research, elaboration of exclusion and inclusion criteria, selection of studies categorization of the selected studies, analysis and interpretation of the results. The research asked the following question: "What impacts did the COVID-19 pandemic have on the mental health of professionals working in the front line?". The studies highlight the consequences of the covid-19 pandemic on the lives of health professionals, pointing out that exposure to an emergency in public health caused occupational risk and psychological suffering due to trauma, professional devaluation, self-isolation and quarantine. This triggered several negative impacts on the mental health of professionals manifesting themselves through symptoms of insomnia, anxiety, anxiety, depression, fear, frustration and post-traumatic stress. It is concluded that, the impacts that the pandemic brought with the arrival of a new virus little known, reached directly in society and reflected in the health professionals who work in the front line, however, support and psychological care can contribute to face the effects of the pandemic on mental health, as well as other alternatives such as training courses to guide the health team in the treatment and coping with psychological problems.
\end{abstract}

Keywords: Coronavirus; Mental health; Health personnel.

\title{
Resumen
}

Se trata de una revisión integradora de la literatura de enfoque cualitativo. Siendo seguida las siguientes etapas de identificación del tema y selección de la investigación, elaboración de los criterios de exclusión e inclusión, selección de los estudios categorización de los estudios seleccionados, análisis e interpretación de los resultados. La investigación tuvo como pregunta orientadora la siguiente pregunta: ¿¿Cuáles son los impactos de la pandemia del COVID-19 en la salud mental de los profesionales que actúan en la línea de frente?". Los estudios destacan las consecuencias de la pandemia del covid-19 en la vida de los profesionales de la salud, al señalar que la exposición a una situación de emergencia en salud pública provocó riesgo ocupacional y sufrimiento psicológico debido al trauma, devaluación profesional, auto aislamiento y cuarentena. Con eso, fue desencadenado diversos impactos negativos en la salud mental de los profesionales manifestándose mediante síntomas de insomnio, angustia, ansiedad, depresión, miedo, frustración y estrés postraumático. Se concluye que, los impactos que la pandemia trajo con la llegada de un nuevo virus poco conocido, golpeó directamente en la sociedad y reflejó en los profesionales de la salud que actúan en la línea de frente, Sin embargo, el apoyo y la acogida psicológica podrán contribuir a enfrentar los efectos de la pandemia en la salud mental, además de otras alternativas como cursos de capacitación para orientar al equipo de salud en el tratamiento y enfrentamiento de problemas psicológicos.

Palabras clave: Coronavirus; Salud mental; Personal de salud. 


\section{Introdução}

Com a chegada da pandemia, de um vírus novo, pouco estudado e conhecido, da Síndrome Respiratória Aguda Grave (SARS-CoV-2), se espalhou rapidamente pelo mundo após o primeiro caso que foi relatado em Wuhan na China. O novo coronavírus foi nomeado de SARS-CoV-2, pelo Comitê Internacional de Taxonomia de Vírus e a doença infectada por esse vírus foi denominada COVID-19 (Lu et al., 2020).

Com a rápida disseminação do vírus, o governo da China emitiu um anúncio público sobre o tratamento e prevenção da doença infecciosa mais grave, o que exigia uma determinação de medidas eficazes para prevenir a transmissão da doença (Lu et al., 2020). Os diversos tipos de coronavírus como a Síndrome Respiratória Aguda Grave (SARS) e a Síndrome Respiratória do Oriente Médio (MERS) e atualmente a COVID-19 causam na humanidade desde resfriados comuns até doenças mais graves levando a complicações e até a morte de um grande contingente de pessoas (Acelas et al., 2021).

No Brasil, ainda existe há incerteza da ocorrência dos primeiros casos, tendo em vista as características dos surgimentos dos sintomas e a rápida e silenciosa propagação da infecção, entretanto foi confirmada a primeira morte por diagnóstico de COVID-19 em 25 de fevereiro de 2020 em São Paulo (Seixas, 2021).

O coronavírus é conhecido por ser transmitido com rápida propagação em comparação com outros coronavírus sendo através de transmissão via gotículas respiratórias e pelo contato direto e indireto por fômites contaminados causando dificuldade no controle do vírus (Acelas et al., 2021). Como é novidade o SARS-CoV-2, existem poucas informações sobre sua disseminação, tratamento e controle com isso é desencadeado nos profissionais de saúde medo e estresse sendo um desagradável estado mental produzido pela percepção de perigo iminente e desconhecido (Fernández et al., 2021).

O surgimento de uma doença súbita e que apresenta um elevado risco de morte promove aos profissionais que estão na linha de frente elevação da pressão psicológica aumentando assim sua exaustão física, aumento da carga horária de trabalho, alta transmissibilidade hospitalar e necessidade de tomada de decisões eticamente difíceis sobre o racionamento dos cuidados e seu bem-estar mental e físico (Santos, 2021).

Com o avanço da pandemia do novo coronavírus, se teve uma sobrecarga nos serviços de saúde em detrimento dos casos suspeitos e confirmados da COVID-19, fazendo com que exista uma grande procura pelos serviços de saúde por demandas relacionadas à Saúde Mental, tendo em vistas nas repercussões negativas que afetam a coletividade, inclusive os profissionais de saúde que estão na linha de frente, provocadas pela pandemia e não podendo ser negligenciadas (Dantas, 2021).

Os profissionais de saúde que atuam na linha de frente a pandemia, enfrentam o isolamento e discriminação social o que os tornam um grupo vulnerável aos sentimentos emocionais complexos e sofrimento psicológico. Com as incertezas sobre o tratamento do vírus, vacina, taxa de transmissibilidade do vírus afetem o nível de ansiedade dos mesmos. Justamente a isso, vivenciam problemas no convívio social e familiar pelo medo de infecção levantando assim diversas emoções negativas (Aksoy et al., 2020).

Com as repercussões mentais nos períodos supracitados, podem se destacar a desesperança, medo exacerbado, medo da morte em si e de pessoas próximas, medo de ser infectado e infectar as outras pessoas, enfrentamento de medidas de isolamento social, ansiedade, sintomas depressivos e de comportamento suicida (Opas, 2016).

Mesmo assim, no contexto de pandemia os profissionais da saúde são convocados e permanecem exercendo seu trabalho, o que fazem com que eles fiquem expostos a possibilidade de contrair o vírus encontrando-se suscetíveis a riscos ocupacionais e a pressão psicológica (Crispim et al., 2020).

É notório que os profissionais de saúde, principalmente os que atuam na linha de frente de combate a COVID-19, estão submetidos a um constante e elevado risco de contágio (Veloso, 2020) e enfrentam, nem sempre, confiáveis condições de trabalho, pois estão em ambientes inseguros, com inadequada infraestrutura, submetidos a uma longa carga horária de trabalho, 
lidando com escassez de Equipamentos de Proteção Individual (EPI) e preocupados com a saúde de seus pacientes (Bezerra et al., 2020).

Assim, considerando a vulnerabilidade dos profissionais de saúde que atuam de forma direta e indireta na assistência e combate ao coronavírus ao confronto com seus recursos físicos e psicológicos. Surge assim a necessidade de responder ao seguinte questionamento: "Quais os impactos a pandemia do COVID-19 trouxeram na saúde mental dos profissionais atuantes na linha de frente?" Diante do exposto o estudo visa evidenciar os principais impactos na saúde mental dos profissionais que atuam na linha de frente a pandemia do COVID-19.

\section{Metodologia}

Trata-se de uma revisão integrativa da literatura de abordagem qualitativa (Ludke et al., 2013). Sendo seguida as seguintes etapas de identificação do tema e seleção da pesquisa, elaboração dos critérios de exclusão e inclusão, seleção dos estudos categorização dos estudos selecionados, análise e interpretação dos resultados (Souza et al., 2010).

Na utilização da estratégia de busca foi utilizado os descritores pré-estabelecidos pelo Descritores em Ciências da Saúde (DECS), sendo esses: “Coronavírus", "Saúde Mental” e "Pessoal da Saúde”. Tendo como pergunta norteadora e tema de interesse: "Quais os impactos a pandemia do COVID-19 trouxe na saúde mental dos profissionais atuantes na linha de frente?". Assim, foi utilizada a base de dados da Literatura Latino Americana e do Caribe em Ciências da Saúde (LILACS).

A consulta ocorreu durante o mês de janeiro de 2022. As versões em português dos descritores compuseram as estratégias de busca a partir do cruzamento: I) Coronavírus AND Saúde Mental AND Pessoal da Saúde. Desta forma, a amostra selecionada entre tantos disponíveis resultou em 103 artigos.

Os critérios de inclusão adotados foram: I) período de publicação entre os anos de 2019 a 2022; e II) estudos clínicos e randomizados e que pudessem responder à questão norteadora a partir da leitura do título e resumo. Os critérios de exclusão envolveram estudados duplicados e que correspondessem a uma revisão integrativa, livros, cartas ao editor e artigos de nota prévia. Em seguida foi selecionado o quantitativo de nove artigos para compor o corpus de análise de artigos elegíveis. A figura I descreve como foi realizada a seleção dos estudos.

A busca e seleção dos artigos foram realizadas e inicialmente identificou-se os estudos que apresentavam duplicidade entre as bases de dados pesquisadas e após isso, foi feita a avaliação do título e resumo. Além disso, foi realizada uma leitura integral dos artigos identificados como potencialmente elegíveis, sendo incluídos no estudo, aqueles que estavam de acordo com os critérios de inclusão e exclusão. 
Figura I: Seleção dos estudos encontrados. Brasil, 2022.

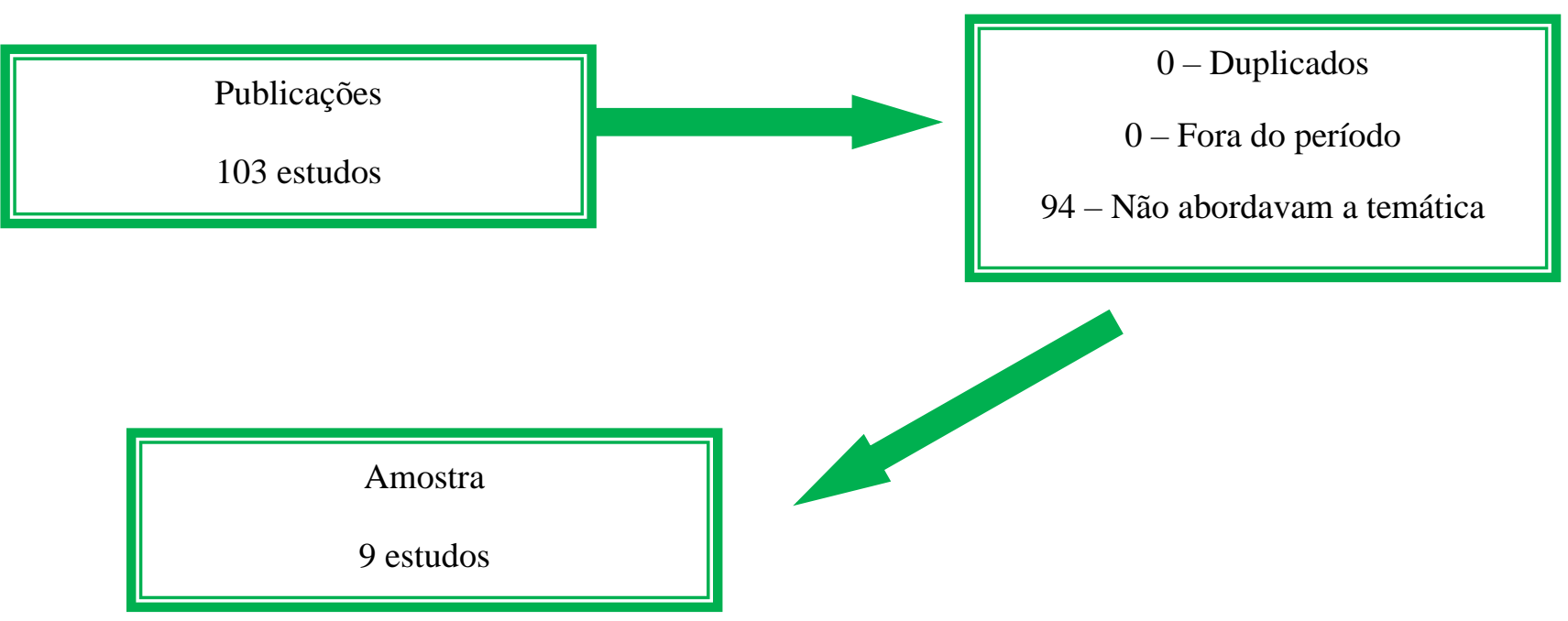

Fonte: Autores (2022).

\section{Resultados e Discussão}

Os estudos mais atuais foram selecionados e agrupados no Quadro 1, contendo informações sobre autoria, ano de publicação, título e resultados. Dessa forma, uma coleta de estudos atualizados proporciona uma discussão na revisão integrativa referente aos principais impactos na saúde mental dos profissionais de saúde atuantes na linha de frente a pandemia do novo coronavírus. No quadro 1 foi elaborado o corpus de análise de pesquisa representado a seguir:

Quadro 1: Corpus de análise de pesquisa. Brasil, 2022.

\begin{tabular}{|c|c|c|}
\hline Autoria e ano de publicação & Título do artigo & Resultados \\
\hline BARRETO, M. S. et al., 2021. & $\begin{array}{c}\text { Pandemia da COVID-19: repercussões } \\
\text { no quociente da família de profissionais } \\
\text { de saúde atuantes em unidades } \\
\text { emergenciais }\end{array}$ & $\begin{array}{c}\text { Em consequência, o isolamento social imposto pelo medo } \\
\text { de contaminação com o novo Coronavirus desencadeou } \\
\text { impactos negativos na saúde mental dos profissionais da } \\
\text { saúde pela impossibilidade de manter contato próximo } \\
\text { com familiares e amigos. Esses impactos manifestaram-se } \\
\text { mediante sintomas de ansiedade, insônia, angústia, } \\
\text { estresse pós-traumático e depressão. }\end{array}$ \\
\hline BENDAU, A. et al., 2021. & $\begin{array}{c}\text { Mental health of health professionals in } \\
\text { the coronavirus (Covid-19) pandemic }\end{array}$ & $\begin{array}{c}\text { Altas proporções de profissionais de saúde relatam } \\
\text { sintomas agudos de ansiedade, depressão, alto estresse } \\
\text { psicológico e insônia no contexto da pandemia de } \\
\text { COVID-19. }\end{array}$ \\
\hline CHEN, C. H. et al., 2021. & $\begin{array}{c}\text { The 2003 SARS experience has a } \\
\text { negative psychological impact on } \\
\text { healthcare workers in the COVID-19 } \\
\text { pandemic: a cross-sectional study }\end{array}$ & $\begin{array}{c}\text { Durante o surto de COVID-19, os profissionais de saúde } \\
\text { da linha de frente com experiência no tratamento da } \\
\text { fARS-CoV-2 indicaram níveis mais elevados de estresse } \\
\text { quelação à carga de trabalho de cuidar de pacientes do } \\
\text { frente sem experiência no tratamento da SARS. }\end{array}$ \\
\hline FAIROZEKHAN, A. T. et al., & $\begin{array}{c}\text { Psychological perceptions and } \\
\text { preparedness during the novel } \\
\text { coronavirus disease - 2019 pandemic } \\
\text { (covid-19) - a multinational cross- } \\
\text { sectional study among healthcare } \\
\text { professionals }\end{array}$ & $\begin{array}{c}\text { Estresse emocional intenso foi relatado pelos } \\
\text { profissionais de saúde quando os colegas são } \\
\text { infectados. Os profissionais da área médica (61,7\%) } \\
\text { apresentaram mais estresse no trabalho em comparação } \\
\text { com os profissionais da área odontológica. }\end{array}$ \\
\hline
\end{tabular}




\begin{tabular}{|c|c|c|}
\hline FERRARI, J. et al., 2021. & $\begin{array}{l}\text { Cuidados em saúde mental ofertados a } \\
\text { profissionais de saúde durante a } \\
\text { pandemia de Covid-19 }\end{array}$ & $\begin{array}{l}\text { Os resultados foram classificados em três categorias: o } \\
\text { estado de saúde mental dos trabalhadores de linha de } \\
\text { frente, apoio oferecido a esses profissionais, e apoio } \\
\text { recebido pelos profissionais de saúde mental para a } \\
\text { realização dessas atividades. A análise indicou que muitos } \\
\text { profissionais, tanto da linha de frente quanto da saúde } \\
\text { mental, referiram sintomas psicológicos desencadeados } \\
\text { ou agravados pela pandemia, preocupações com a } \\
\text { possibilidade de contaminar alguém próximo, } \\
\text { invisibilidade das necessidades em saúde mental da } \\
\text { população, e a falta de investimento nesse setor }\end{array}$ \\
\hline LUZ, D. C. R. P. et al., 2021. & $\begin{array}{l}\text { Burnout e saúde mental em tempos de } \\
\text { pandemia de COVID-19: Revisão } \\
\text { sistemática com metanálise }\end{array}$ & $\begin{array}{c}\text { Diante da atual realidade ocorreu uma elevação na carga } \\
\text { de trabalho e nos cuidados de prevenção, acentuando a } \\
\text { exaustão física e emocional. É importante frisar que esses } \\
\text { profissionais tendem a se descuidar da sua saúde mental e } \\
\text { passam por desafios como o enfrentamento do } \\
\text { distanciamento social dos seus próprios familiares, medo, } \\
\text { angustia e cansaço }\end{array}$ \\
\hline $\begin{array}{l}\text { MALAQUIAS, T. S. M. et al., } \\
2021 .\end{array}$ & $\begin{array}{l}\text { Effects of the covid-19 pandemic on } \\
\text { health professionals: systematic review } \\
\text { protocol }\end{array}$ & $\begin{array}{c}\text { Espera-se que identifique estudos que apontam para a } \\
\text { prevalência de transtornos mentais comuns e sinais e } \\
\text { sintomas físicos mentais apresentados por profissionais de } \\
\text { saúde que prestaram atendimento direto a pacientes } \\
\text { infectados com SARS-CoV-2 }\end{array}$ \\
\hline $\begin{array}{l}\text { MARTINOLA, D. R. H. et al., } \\
2021 .\end{array}$ & $\begin{array}{l}\text { Vulnerability in mental health of health } \\
\text { personnel to COVID-19 }\end{array}$ & $\begin{array}{c}\text { As áreas mais afetadas foram as cognitivas e as afetivas, } \\
\text { expressando mais intensidade nos sintomas relacionados } \\
\text { às dificuldades para concentração, tristeza e ansiedade. A } \\
\text { existência de uma maior vulnerabilidade na saúde mental } \\
\text { do pessoal antes de começar a trabalhar com pacientes } \\
\text { suspeitos e positivos para COVID-19, expressa a } \\
\text { necessidade de manter a etapa de Prevenção Primária } \\
\text { como parte do Protocolo de Gestão de Segurança } \\
\text { Psicológica. }\end{array}$ \\
\hline PEDROSO, R. M. et al., 2021. & $\begin{array}{l}\text { Síntomas de ansiedade y depresión em } \\
\text { personal de salud que trabaja com } \\
\text { enfermos de COVID-19 }\end{array}$ & $\begin{array}{c}\text { Os participantes relataram nervosismo, cansaço, e a } \\
\text { principal preocupação foi a morte do paciente. Além } \\
\text { disso, indicaram que o período ideal de cuidados } \\
\text { contínuos de pacientes com COVID-19, para evitar o } \\
\text { aparecimento de problemas psicológicos sintomas foi de } 7 \\
\text { dias. }\end{array}$ \\
\hline
\end{tabular}

Fonte: Autores (2022).

A pandemia faz com que aumente o nível de medo e estresse entre os profissionais resultando assim em comportamentos de evitação, seja epidêmico ou pandêmico os mesmos estão na vanguarda de cada surto e acabam arriscando suas vidas, não estando apenas sob tremendo estresse, mas também podem sofrer consequências psicológicas de longo prazo pós-pandemia (Fairozekhan et al., 2021).

Com os impactos causados pela pandemia do COVID-19, constituindo-se em um evento vital de enorme significado causando impacto emocional nos profissionais de saúde que atuam na linha de frente. Foi identificado nos estudos que o COVID-19 modificou a vida cotidiana, estilo de vida e o trabalho dos mesmos, visto que a principal preocupação dos profissionais é que o paciente desenvolva complicações e venha a falecer, sendo o vírus determinado pelas características da doença como a alta agressividade e mortalidade principalmente em pessoas de idade avançada e que possuem fatores de riscos associados (Pedroso, Izquierdo \& David, 2021).

Estudos mostram a depressão como um transtorno mental comum, que é caracterizado pela presença de tristeza, sentimento de culpa ou falta de autoestima, distúrbios do sono ou apetite, sensação de cansaço, falta de concentração e perda de interesse ou prazer. Assim, trabalhar na linha de frente reflete um fator de risco e suas descobertas levantam preocupações sobre o bem-estar psicológico de profissionais envolvidos nos cuidados (Pedroso et al., 2021). 
Também foram encontrados que a jornada de trabalho, medo do contágio, pressão dos cuidados de saúde e a necessidade de tomar decisões possuem um impacto decisivo na vida das pessoas, especialmente em profissionais que trabalham em unidades de cuidados intensivos. Com isso, os profissionais têm aumentado sua carga de trabalho, devido ao grande volume de pacientes com sinais e sintomas de COVID-19, portanto a sobrecarga e fadiga podem desafiar ainda mais o sistema imunológico e aumentar a suscetibilidade ao vírus (Pedroso et al., 2021).

Em outro estudo foi encontrado sintomas somáticos, como problemas gastrointestinais sendo de maior intensidade a diarreia, vômito e salto epigástrico, considerados como fatores físicos associados a um alto impacto psicológico nos sintomas de depressão e ansiedade. Além disso, a presença de sintomas relacionados a mudanças de comportamento como agressividade, apatia e irritabilidade foram significativos devido a exposição a situações ameaçadoras (Martinola et al., 2021).

Com a pandemia do COVID-19, é comum que os profissionais que estão atuando na linha de frente possuam sentimentos de apreensão e horror, devido ao aumento da preocupação com os amigos e familiares, além de apresentarem níveis mais elevados de stress devido a possibilidade aumentada de transmissibilidade infectocontagiosa, sendo assim submetidos a um momento de fragilidade emocional (Luz et al., 2021).

O elevado número de profissionais contaminados e afastados do ambiente laboral leva à uma sobrecarga de trabalho ainda maior para as equipes de saúde, contribuindo de forma relevante para o esgotamento físico para equipe atuante. O estresse ocupacional tem sido um fator importante de indicador de exaustão psíquica relacionado ao enfrentamento da pandemia gerando assim algumas incertezas fazendo com que se tenha uma reflexão sobre a saúde mental dos profissionais (Luz et al., 2021).

Alguns profissionais de saúde que atuavam no combate ao coronavírus afirmam que houve um agravamento de situações e sintomas pré-existentes e assim intensificados pela pandemia como a violência doméstica, ideação suicida, automutilação, problemas relacionados com a equipe, uso abusivo de álcool e conflitos conjugais. Além disso, também foi relatado que sintomas depressivos, ansiedade, angústia e sensação e impotência foram desencadeados pelo evento da pandemia especialmente no que se refere à falta de informações precisas das autoridades como também aos rígidos protocolos de isolamento e higiene (Ferrari et al., 2021).

Situações de crise, requeiram mudanças e reestruturação de vida proporcionando aos profissionais na linha de frente o desenvolvimento de sintomas de estresse, reações físicas, mudança no comportamento e no nível de atividade, com isso as condições de saúde mental pré-existentes são apontadas como riscos psicossociais que podem aumentar com a pandemia (Ferrari et al., 2021).

Perturbações emocionais e mentais foram frequentemente encontradas nos profissionais de saúde. Nesse estudo, foi encontrado que $43 \%$ dos participantes sentiam que a força de trabalho era inadequada para lidar com o aumento da demanda, relatando também sobrecarga mental e psicológica dos trabalhadores da saúde, suas preocupações relacionadas ao trabalho e possíveis métodos de restauração, necessitando assim cuidar de si mesmos (Fairozekhan et al., 2021).

Os estudos destacam as consequências da pandemia do covid-19 na vida dos profissionais de saúde, ao apontar que a exposição a uma situação de emergência em saúde pública provocou risco ocupacional e sofrimento psicológico devido ao trauma, desvalorização profissional, auto isolamento e quarentena. Com isso, desencadeou diversos impactos negativos na saúde mental dos profissionais manifestando-se mediante a sintomas de insônia, angústia, ansiedade, depressão, medo, frustração e estresse pós-traumático (Barreto et al., 2021).

Os profissionais atuantes na linha de frente relatam que os Equipamentos de Proteção Individual (EPI), é necessário para conter a propagação do vírus ao lidar com casos confirmados ou suspeitos, os profissionais relatam que usam um conjunto completo de equipamentos a fim de reduzir a transmissibilidade, contudo tem experimentado estados de fadiga e tensão aumentando assim a dificuldade no seu trabalho tornando mais propensos ao esgotamento (Chen et al., 2021). 


\section{Conclusão}

É notório os impactos que a pandemia do novo coronavírus trouxe para a sociedade e para os profissionais de saúde atuantes na linha de frente ao combate à doença. Assim, é preciso que se tenha auxílio da psicologia para que possa trazer contribuições importantes e relevantes para encarar os efeitos da COVID-19 na saúde mental, tendo assim uma emergência de saúde pública de grande magnitude.

As implicações à saúde mental dos profissionais da saúde incluem estresse emocional, perda da qualidade do sono, esgotamento, preocupação, ansiedade e medo. Decorrente do contexto pandêmico, fazendo com que os mesmos enfrentem condições laborais precárias e exposições a longas jornadas de trabalho.

Assim, é preciso que se tenha um plano de intervenção psicológica, com oferta de cursos online para orientar a equipe de profissionais de saúde no tratamento de problemas psicológicos comuns, tendo assim supervisão para resolução de problemas psicológicos ou quaisquer dificuldades que poderá surgir, sendo baseada nas necessidades dos profissionais de saúde sendo adaptada a diversas culturas, origens, crenças religiosas e preferências pessoais. Além de, reforçar ainda mais o âmbito cientifico para novos estudos acerca do tema.

\section{Referências}

Acelas, A. L. R., Gential, D. Y., \& Montañez, W. C. (2021). Correlacíon entre diagnósticos, resultados e intervenciones de enfermeira en el cuidado al paciente hospitalizado por COVID-19. Revista Cuidarte, 12(1944). https://revistas.udes.edu.co/cuidarte/article/view/1944

Aksoy, Y. E., \& Koçak, V. (2020). Psychological effects of nurses and midwives due to covid-19 outbreak: the case of Turkey. Archives of psychiatric nursing, 34(5), 427-433. https://pubmed.ncbi.nlm.nih.gov/33032769/

Barreto, M. S., Hipolito, A. B. L., Hipolito, M. A. L., Lise, F., Radovanovic, C. A. T. \& Marcon, S. S. (2021). Pandemia da COVID-19: repercussões no quociente da família de profissionais de saúde atuantes em unidades emergenciais. Esc. Anna Nery Rev Enferm, 25 , e20210064. https://pesquisa.bvsalud.org/portal/resource/pt/biblio-1281638

Bendau, A., Strohle, A. \& Petzold, M. B. (2021). Mental health of health professionals in the coronavirus (Covid-19) pandemic. Adv. Exp. Med. Biol, 1318, 737-757. https://pubmed.ncbi.nlm.nih.gov/33973209/

Bezerra, G. D., Sena, A. S. R., Braga, S. T., Santos, M. E. N., Correia, L. F. R., Clementino, K. M. F., Carneiro, Y. V. A., \& Pinheiro, W. R. (2020). O impacto da pandemia por COVID-19 na saúde mental dos profissionais de saúde: revisão integrativa. Revista Enfermagem Atual In Derme, 93, e020012. https://www.revistaenfermagematual.com.br/index.php/revista/article/view/758\#: :text=Os\%20estudos\%20trazem\%20como\%20foco,com\%20sa\%C3\%BAde $\% 20 \mathrm{f} \% \mathrm{C} 3 \%$ ADsica $\% 20 \mathrm{e} \% 20$ mental.

Chen, C. H., Yang, P. H., Kuo, F. L., Yeh, I. J. \& Su, C. Y. (2021). The 2003 SARS experience has a negative psychological impact on healthcare workers in the COVID-19 pandemic: a cross-sectional study. São Paulo Med https://www.scielo.br/j/spmj/a/3ptBLtSxV9VV7XZmxM5ny9L/?lang=en\&format=pdf

Crispim, D., Silva, M. J. P., Cedotti, W., Câmara, M. \& Gomes, S. A. Comunicação difícil e Covid-19: Recomendações práticas para comunicação e acolhimento em diferentes cenários da pandemia. BDENF, 31. https://pesquisa.bvsalud.org/portal/resource/pt/biblio-1103572

Dantas, E. S. O. (2021). Saúde Mental dos profissionais de saúde no Brasil no contexto da pandemia por COVID-19. Interface, 25(1), e200203. https://www.scielo.br/j/icse/a/rCWq43y7mydk8Hjq5fZLpXg/\#

Fairozekha, A. T.Mohamed, S., Mohammed, F., Kumaresan, R., Bugshan, A. S. M., Sudeep, C. B., Moidin, S., \& AlMoumen, S. A. (2021). Psychological perceptions and preparedness during the novel coronavirus disease - 2019 pandemic (covid-19) - a multinational cross-sectional study among healthcare professionals. Brazilian Dental Journal, 32(4), 116-126. https://www.scielo.br/j/bdj/a/RDp9xHgrt7qVpXXCJZxVKYS/\#

Fernández, I. A. M., Moreno, S. C. Díaz, L. C., Torees, R. M. G., Fernández, J. A. M. \& Martírez, E. K. H. Fear, Stress, and Knowledge regarding COVID-19 in nursing students and recente graduates in México. Invest. Educ. Enferm, 39(1). https://pubmed.ncbi.nlm.nih.gov/33687809/

Ferrari, J., Renck, B. \& Goergen, P. (2021). Cuidados em saúde mental ofertados a profissionais de saúde durante a pandemia de Covid-19. Revista Brasileira de Psicoterapia, 23(1), 127-142. https://cdn.publisher.gn1.link/rbp.celg.org.br/pdf/v23n1a11.pdf

Lu, W., Wang, H., \& Lin, Y. (2020). Estado psicológico da força de trabalho médica durante a pandemia de COVID-19: um estudo transversal. Psychiatry Research, 288. https://www.sciencedirect.com/science/article/pii/S0165178120305850?via\%3Dihub

Luz, D. C. R. P., Campos, J. R. E., Bezerra, P. O. S., Campos, J. B. R., Nascimento, A. M. V. \& Barros, A. B. (2021). Burnout e saúde mental em tempos de pandemia de COVID-19: Revisão sistemática com metanálise. http://revistas.mpmcomunicacao.com.br/index.php/revistanursing/article/view/1540 
Research, Society and Development, v. 11, n. 3, e18011326491, 2022

(CC BY 4.0) | ISSN 2525-3409 | DOI: http://dx.doi.org/10.33448/rsd-v11i3.26491

Malaquias, T. S. M., Okubo, C. V. C., Rossaneis, M. A., Aroni, P., Malaquias, A. G., \& Haddad, M. C. F. L. (2021). Effects of the covid-19 pandemic on health professionals: systematic review protocol. Online Brazilian Journal of Nursing, 20: e20216520. http://www.objnursing.uff.br/index.php/nursing/article/view/6520/pdf-pt

Martinola, D. R. H., Jover, M. V. G., Nápoles, M. E. S., Rodriguez, K. F. B., Medina, T. A. H., \& Alvarez, A. K. G. (2021). Vulnerability in mental health of health personnel to COVID-19. Revista Brasileira de Psicoterapia, 23(2), 79-88. https://cdn.publisher.gn1.link/rbp.celg.org.br/pdf/v23n2a09.pdf

Organizacíon Mundial de La Salud. (2016). Organización Panamericana de la Salud. Prevención de la conducta suicida. OPAS

Pedroso, R. M., Izquierdo, M. \& David, E. (2021). Síntomas de ansiedade y depresión em personal de salud que trabaja com enfermos de COVID-19. Int. J. Med Surg Sci, 8(1), 1-12. https://pesquisa.bvsalud.org/portal/resource/pt/biblio-1151581

Santos, B. M. P. (2021). A face feminina na linha de frente contra a pandemia de covid-19. Nursing, 24(275), 5450-5483. https://pesquisa.bvsalud.org/controlecancer/resource/pt/biblio-1223594?src=similardocs

Seixas, C. T. (2021). A crise como potência: os cuidados de proximidade e a epidemia pela COVID-19. Interface, 25(1), 57-62. http://dx.doi.org/10.1590/interface.200379

Souza, M. T., Silva, M. D. \& Carvalho, R. Revisão integrativa: o que é e como fazer? Einstein. 8, 102-6. https://doi.org/10.1590/S1679-45082010RW1134.

Veloso, R. S. (2020). Burnout nos profissionais de saúde durante a pandemia covid-19. Dissertação - Faculdade de Psicologia e de Ciências da Educação. 